\title{
Polarized fusion: can polarization help to increase the energy output of fusion reactors?
}

\section{R. Engels*}

Institute for Nuclear Physics, Forschungszentrum Jülich, Wilhelm-Johnen-Str. 1, 52428 Jülich, Germany

E-mail: r.w.engelsefz-juelich.de

\begin{abstract}
For more than 50 years it has been discussed to increase the gain of nuclear fusion reactors with the use of polarized fuel. For example, the total cross sections of the most interesting fusion reactions $d+t \rightarrow{ }^{4} \mathrm{He}+n$ or ${ }^{3} \mathrm{He}+d \rightarrow{ }^{4} \mathrm{He}+p$ are increased by $50 \%$ if the spins of both incoming particles are aligned. This effect will increase the energy output of a fusion reactor more than linearly, e.g. by a factor 4 . However, before polarized fuel can be used for energy production in the different types of reactors, a number of questions must be answered. In this contribution we give an overview on our various activities in this field of research.
\end{abstract}

XVth International Workshop on Polarized Sources, Targets, and Polarimetry,

September 9-13, 2013

Charlottesville, Virginia, USA

${ }^{*}$ Speaker. 


\section{Introduction}

The idea to use nuclear polarized particles as fuel for future thermonuclear fusion reactors has existed since many years as it was discussed in a recent paper [1]. For magnetic confinement as well as for inertial fusion the total fusion cross section can be increased by a significant factor. Especially for the dominant nuclear fusion reactions $d+t \rightarrow{ }^{4} \mathrm{He}+n+17.58 \mathrm{MeV}$ and $d+{ }^{3} \mathrm{He} \rightarrow{ }^{4} \mathrm{He}+p+$ $18.34 \mathrm{MeV}$, a factor close to 1.5 is expected in the energy range below $100 \mathrm{keV}$. The main reaction channel in both cases is a s-wave dominated $J=3 / 2^{+}$resonance. Therefore, the fusion of the deuteron $(J=1)$ and the triton $(t)$ or ${ }^{3} \mathrm{He}$ (both $J=1 / 2$ ) with anti-parallel orientation of the nuclear spins is very weak and only $2 / 3$ of the unpolarized interaction processes lead to fusion. With the spins of both reaction partners aligned this suppression can be excluded. Furthermore, the polarization of the projectiles allows one to control the ejectile trajectories. Due to the s-wave dominance at plasma energies, the differential cross section in the center-of-mass system depends only weakly on the emission angle of the ejectiles. The transformation into the laboratory system, however, results in an increased forward and backward cross section. This asymmetry can be increased or decreased with the use of polarized fuel. This allows one to control the energy transfer from the plasma to the reactor wall or to concentrate the neutron flux to defined wall areas. These facts are undoubted and they were experimentaly confirmed in 1971 [2].

The energy gain of a fusion reactor increases stronger than linearly with the increase of the fusion cross section at a fixed plasma temperature. Increase of the cross section leads to a higher energy density, i.e., an increase of the plasma temperature, which is connected with a higher fusion cross section. It has been shown that for a laser-induced inertial fusion reactor the energy gain can be increased by a factor 4, when polarized fuel is used [3]. As discussed in Ref. [3], the use of polarized fuel would also allow one to reduce the laser power by $25 \%$ and to maintain the energy gain due to the increase of the cross section compared with the unpolarized case. For a Tokomak reactor such calculations do not exist thus far. In any case, however, higher fusion-reaction rates will lead to increased heating of the plasma by, e.g., the higher density of thermalized $\alpha$ particles. Higher temperatures in the equilibrium on their turn will increase the total cross section. Such calculations should be performed for the specific configurations of magnetic-confinement reactors.

\section{Open Questions}

Before polarized fuel can be considered to be used in future thermonuclear reactors of different types, a long list of questions must be answered.

\subsection{Fundamental Physics: What about the $d-d$ reactions?}

The increase of the fusion cross section for the above-mentioned $d-t$ and $d-{ }^{3} \mathrm{He}$ reactions by using polarized fuel is out of doubt. For the reactions (1) $d+d \rightarrow t+p$ and (2) $d+d \rightarrow{ }^{3} \mathrm{He}+n$, however, which also occur in a plasma containing deuterium, the situation is unknown. For both reactions, Fig. 1 shows the predictions for the energy dependence of the quintet-suppression factor, the ratio of the total cross section $\sigma_{1,1}$ for parallel deuteron-spin orientation (quintet state) and the unpolarized total cross section $\sigma_{0}$. In the energy range of a Tokomak reactor $(30-50 \mathrm{keV})$ the calculations indicate the possibility that the polarized fusion cross section of reaction (1) may lie 


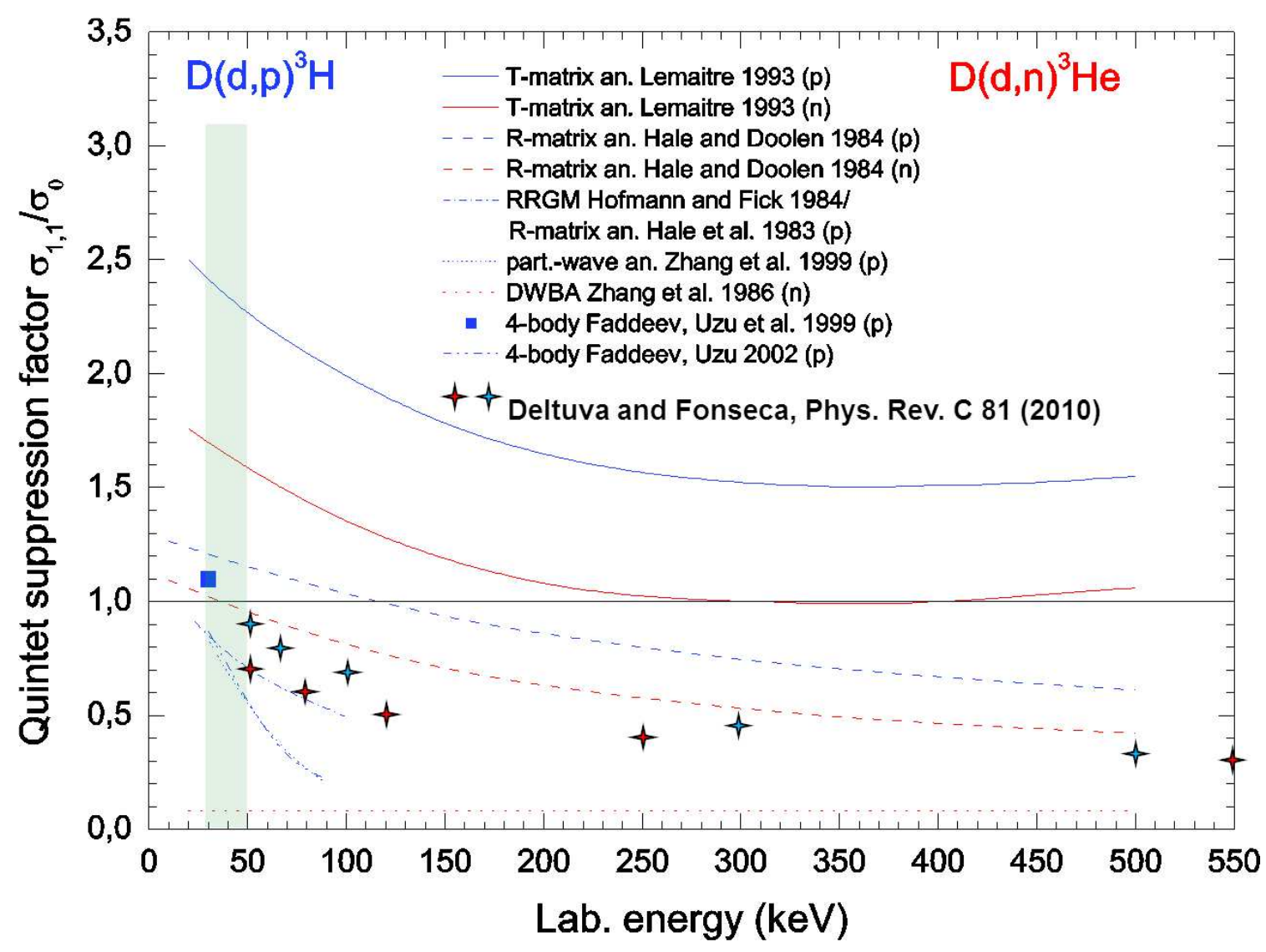

Figure 1: Theoretical predictions for the ratio of the total cross section for parallel deuteron spins $\sigma_{1,1}$ and the unpolarized case $\sigma_{0}$ in the energy range up to $550 \mathrm{keV}$. Most of the references are found in the publication of Deltuva and Fonseca ([5]).

by a factor of 2.5 higher than the unpolarized one. Furthermore, the predictions (in the figure the energy-independent dotted line) indicate that the polarized cross section of reaction (2), i.e., neutron production, may be suppressed by a factor 10 compared to that of reaction (1). This would reduce the rate of wall-material damage by the fast neuterons. The theoretical study of the $d-d$ reactions is complicated because of the strong influence of $p$ and $d$ waves. A measurement of the spin-correlation coefficients and the quintet-suppression factor is the stringent way to discriminate between the theoretical predictions. In a dedicated experiment, under preparation at the Petersburg Nuclear Physics Institute (PNPI) in Gatchina, Russia, it is planned to measure the spin-correlation coefficients of the $d-d$ reactions. The setup is shown in Fig. 2. A polarized atomic beam source (ABS), formerly used at the University of Ferrara, Italy, will be used to produce a polarized deuterium jet. Its vector and tensor polarization will be measured with a Lamb-shift polarimeter (LSP), used before at Universität zu Köln, Germany, in the SAPIS project. The polarized deuteron beam (energy $\leq 32 \mathrm{keV}$ ) will be generated by the polarized ion source (POLIS), contributed by KVI in Groningen, The Netherlands. It will be tuned with the LSP form the POLIS project. The longterm stability will be controlled with a polarimeter, built in Jülich and making use of the known analyzing powers of the $d-d$ fusion reactions [4]. 


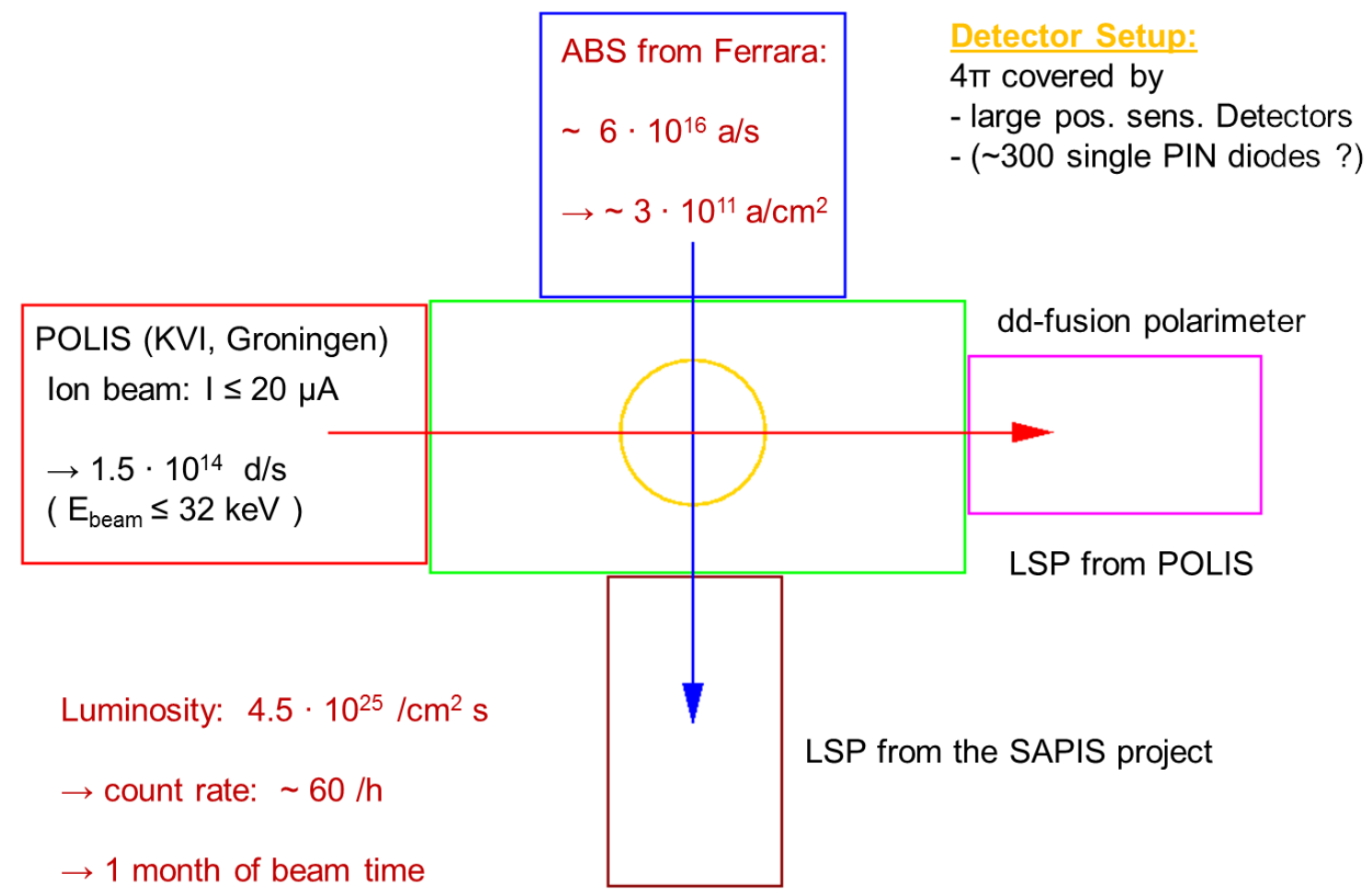

Figure 2: Scheme of the setup for $\vec{d}-\vec{d}$ fusion experiments with $E_{\vec{d}} \leq 32 \mathrm{keV}$ under preparation at PNPI, Gatchina, Russia. The $\vec{d}$ beam from the POLIS ion source will cross the $\vec{D}$ beam delivered by the polarized atomic beam source (ABS). The polarization of both beams are measured by Lamb-shift polarimeters (LSP) and a polarimeter making use of the known analyzing powers of the $\vec{d}-\vec{d}$ fusion reactions. The expected luminosity and count rate for a $30 \mathrm{keV}$ beam from POLIS are given.

Besides the effect of the nuclear spin orientation to the fusion cross sections it is thought possible to study the electron-screening effect. The increase of the fusion cross section at energies $<20 \mathrm{keV}$ due to the shielding of the nuclear charges by the $\vec{D}$ electron is well known in astrophysics [6]. The eventual dependence of this effect on the electron spin orientation relative to the nuclear spin orientations can be studied with the setup, too.

\subsection{Can the polarization survive in the plasma?}

In 1982 it was predicted that the lifetime of polarized fuel in a Tokomak reactor should be long enough to influence the total cross section in a fusion plasma [7]. This hypothesis has not yet been verified experimentally. Now an experiment is being planned to study the maintenance of the proton and deuteron polarization in $\overrightarrow{\mathrm{H}} \overrightarrow{\mathrm{D}}$ ice by measuring the influence of the laser pulses on the cross section of the reactions $\vec{d}(\vec{d}, n)^{3} \mathrm{He}$ and $\vec{d}(\vec{p}, \gamma) t$ [8]. Furthermore, in collaboration with groups from Universität Düsseldorf and Research Center Jülich it is planned to investigate the polarization maintenance of a ${ }^{3} \mathrm{He}$ gas during full ionization and acceleration to the lower $\mathrm{MeV}$ region in the electric and magnetic field of a strong laser. The results are expected to yield information on the applicability of polarized fuel in the laser-induced fusion. 


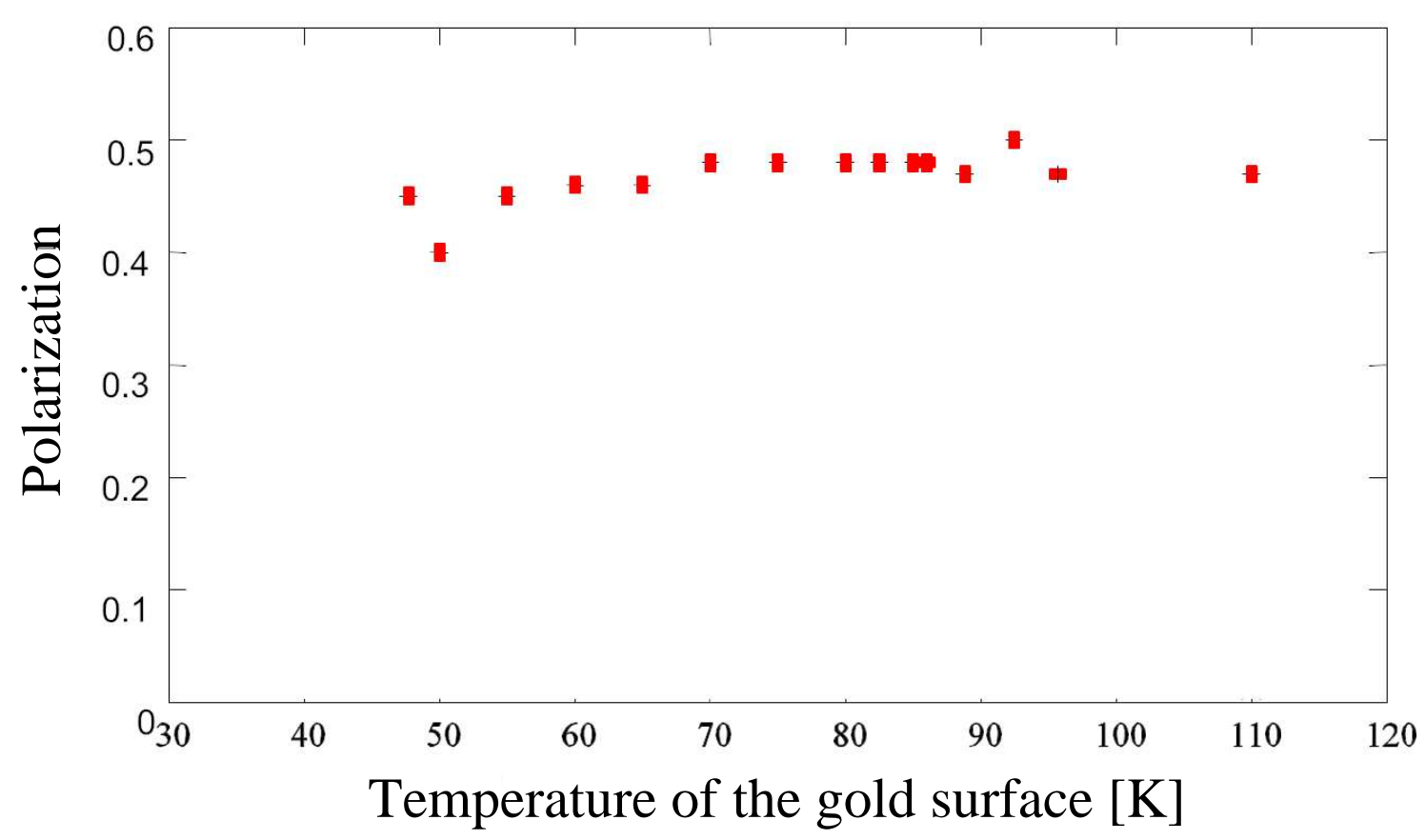

Figure 3: Polarization of protons in hydrogen molecules resulting from recombination of polarized atoms on a gold surface at $0.28 \mathrm{~T}$ as function of the wall temperature.

\subsection{How to get enough polarized fuel?}

In order to use polarized particles to increase the energy gain of a fusion reactor, sufficient polarized fuel has to be provided. This seems not to be a problem for polarized ${ }^{3} \mathrm{He}$ and ${ }^{3} \mathrm{H}$. Both can be produced by optical pumping. As an additional advantage, due to the shielding by the two spinsaturated electrons in wall collisions the gas of polarized ${ }^{3} \mathrm{He}$ can be stored. Polarized deuterium atoms, however, are produced by atomic beam sources and this limits the production rate to $\sim 10^{17}$ atoms per second. Polarized atomic deuterium, ${ }^{2} \mathrm{H}$, like tritium, ${ }^{3} \mathrm{H}$, with one electron only cannot be stored. It has been shown, however, that the nuclear polarization of hydrogen and deuterium can be partially maintained in recombined molecules. The boundary conditions and the degree of polarization maintenance are investigated by a collaboration of groups from PNPI Gatchina, Russia, from Universität zu Köln, and from Research Center Jülich. A dedicated experimental setup was developed enabling the installation of a gas-storage cell with different wall coatings to study the recombination process as function of the wall temperature in the magnetic field of $\leq 1 \mathrm{~T}$ produced by a superconducting coil. The atoms and recombined molecules are ionized by electron impact and the ions are extracted from the cell. The polarization of the protons or deuterons from atoms and from the recombined $\mathrm{H}_{2}$ or $\mathrm{D}_{2}$ molecules is measured with a Lambshift polarimeter [9]. Figure 3 shows a typical result for recombined hydrogen molecules in a cell with a gold surface in a magnetic field of $0.28 \mathrm{~T}$ as a function of the wall temperature. Around $50 \%$ of the atomic nuclear polarization is conserved in the hydrogen molecules. In the meantime it was shown that recombination on Fomblin at $100 \mathrm{~K}$ allows a polarization conservation during recombination of 
more than $90 \%$ for hydrogen. For deuterium at least $50 \%$ of the initial atomic polarization was preserved during the first attempts.

In a next step, these polarized molecules possibly can be collected and stored for a proof-ofprinciple experiment at a fusion reactor.

\section{Conclusion}

The option to increase the energy gain of future fusion reactors by the use of polarized fuel has to be investigated by experimental studies to verify existing calculations. The chance to increase the energy output of future fusion reactors with the use of polarized fuel should not be missed. The described experiments, already performed or planned, are first steps to make use of this possibility.

\section{References}

[1] H. Paetz gen. Schieck, Eur. Phys. J. A. 44 (2010) 321.

[2] Ch. Leemann et al., Helv. Phys. Acta. 141 (1971).

[3] M. Temporal et al., Nucl. Fusion 52 (2012) 103011.

[4] L. Kröll, A dd nuclear-reaction Polarimeter for the polarized Fusion Project, Diploma-Thesis, University of Aachen (2010).

[5] A. Deltuva and A.C. Fonseca, Phys. Rev. C 81 (2010) 054002.

[6] F. Raiola et al., Eur. Phys. J. A 13 (2002) 377.

[7] R.M. Kulsrud et al., Phys. Rev. Lett. 49 (1982) 1248.

[8] J.-P. Didelez and C. Deutsch, Proc. $19^{\text {th }}$ Int. Spin Physics Symp., J. of Phys.: Conf. Series. 295 (2011) 012169.

[9] R. Engels et al., Polarized Hydrogen/Deuterium molecules: a new option for polarized targets?, contribution to this conference. 